\title{
Implementing a Signal Integrity Course in Undergraduate Education
}

\section{Dr. Aldo Morales, Pennsylvania State University, Harrisburg}

Dr. Aldo Morales was born in Tacna, Peru. Dr. Morales earned his B.S. in Electronic Engineering, with distinction, from Northern University (now University of Tarapaca), Arica, Chile. He has an M.Sc. Ph.D. in electrical and computer engineering from University of Buffalo, The State University of New York at Buffalo, Buffalo, NY. Currently, he is a professor of electrical Engineering at Penn State Harrisburg. Dr. Morales was the PI for a 3-year Ben Franklin Technology Partners Grant that established the "Center of Excellence in Signal Integrity" at Penn State Harrisburg. He was a co-author for the Best Poster Paper Award at the IEEE International Conference on Consumer Electronics 2007, Las Vegas, Nevada, for the paper "Transmitter Pre-emphasis and Adaptive Receiver Equalization for Duobinary Signaling in Backplane Channels". In addition, of Best Paper Award at the IEEE Asia Pacific Conference on Circuits and Systems 96, Seoul, Korea, for the paper "Basis Matrix Representation of Morphological Filters with N-Dimensional Structuring Elements".

\section{Dr. Sedig Salem Agili, Pennsylvania State University, Harrisburg}

Sedig S. Agili received his BS, MS, and Ph.D. in Electrical and Computer Engineering from Marquette University in 1986, 1989, and 1996, respectively. Currently he is a Professor of Electrical Engineering teaching and conducting research in signal integrity of high-speed electrical interconnects, electronic communications, and fiber optic communications. He has authored numerous research articles which have been published in reputable peer refereed journals and conference proceedings. He is the Co-director for The Center of Excellence in Signal Integrity at Penn State Harrisburg. He was honored by the Institute of Electrical and Electronics Engineers (IEEE) with Best paper award at the IEEE International Conference on Consumer Electronics 2007, Las Vegas, Nevada, for the paper " Transmitter Pre-emphasis and Adaptive Receiver Equalization for Duobinary Signaling in Backplane Channels." He was also honored by DesignCon for 2013 Best Paper Award Finalist, for the paper "A Rapid Prototyping of FPGA - Based Duobinary Transmitter/Receiver for High Speed Electrical Backplane Transmission," Santa Clara, CA, January 2013. He received the 2010 Technical Achievement Award from the Central Pennsylvania Engineers Week Council. He is a senior member of the Institute of Electrical and Electronic Engineers and a full member of Sigma Xi. 


\section{Implementing a Signal Integrity Course in Undergraduate Education ${ }^{1}$}

Signal integrity has been identified as one of the key areas for scientific education and research at the national level. Nationally, few universities offer courses in signal integrity (SI), and there are none in the immediate area. Therefore, there is a critical need for signal integrity training and support. This is especially important in the Harrisburg metropolitan area given the high concentration of electrical connector companies.

In this paper, the authors describe the implementation of a signal integrity course that include up-todate topics, modern laboratory experiences and speakers from industrial settings. The topics include: basics of simulation tools to visualize time and frequency domain signals as well as transmission line modeling using PSPICE; introducing a Time Domain Reflectometer as a fundamental tool in signal integrity; crosstalk, including practical examples of where and how it occurs. The following topic is using a Vector Network Analyzer (VNA): this is another critical instrument used by the signal integrity community. The use of this equipment requires performance of the following tasks: calibration, frequency range setup, average setup, and collection of Sparameter data using a VNA. Another critical topic is understanding digital communication channel behavior by observing an eye diagram, including how to obtain and analyze an eye diagram using a modern Bit Error Rate Tester. In addition, a high frequency structural simulator is used to obtain Sparameters for different communication structures. Finally, the authors invited a guest speaker from an industry nearby to talk about practical applications in the SI field. The expected outcome is to produce graduates with hands-on signal integrity experience, who will transfer to the workplace with competitive skills and who will be positioned to extend their academic goals in graduate school.

The authors will also make the course content available to other programs, universities and nearby community colleges. Moreover, the authors intend to align signal integrity course with the extensive electrical connector business in the Harrisburg area.

\section{Introduction}

As the speed of new digital designs are pushed into the gigabit ranges, interconnects performance becomes the key factor in enabling reliable system operation. In order to ensure reliable operation at high data rates, signal integrity has to be addressed ${ }^{1-8}$. Signal integrity means that the signal is unimpaired with regard to its functionality ${ }^{3}$. In a system with long interconnects, there are several elements that can impair the signal such as coupling capacitances, ground capacitances, mutual inductances, self-inductances, and wire resistances. These parasitic elements can produce data losses, crosstalk, jitter, and time delays that can significantly degrade system performance and reliability. Electrical connector companies are now expected to design components for data rates up to the order of 40 gigabits per second; under these constraints, they face increased pressure to ensure that these components meet the signal integrity requirements. For engineers, it is important to detect signal integrity problems at the early design cycle to avoid recall of products. The designer needs to understand the

\footnotetext{
${ }^{1}$ The Authors thank National Science Foundation award \# 1429941 for their support.
} 
characteristics of the materials and interconnects layout to obtain models that can be simulated and verified before production.

Our research indicates that, nationally, there are few universities that offer courses in signal integrity at the undergraduate level, and none are in the immediate surrounding area. In a recent ASEE paper, a signal integrity was proposed by Rose Hulman Institute of Technology faculty ${ }^{9-10}$ to be implemented at their institution. At other institutions, signal integrity modules have been incorporated in existing courses ${ }^{11-12}$. To the authors' knowledge, there have been no reports of the outcomes of the deployed course. Having signal integrity experiences are of critical importance since the Harrisburg metropolitan area has one the highest concentration of connector companies in the world. Note that one of the problems with a signal integrity experience is that the type of equipment, such a high speed sampling oscilloscope and advanced vector network analyzer, that are typically used in the laboratory, tends to be very expensive and beyond the standard laboratory equipment in an EE undergraduate program. The authors were able to leverage several sources i.e, local, state and federal, to build the signal integrity laboratory. Currently, the EE program curriculum at Penn State Harrisburg does not offer courses in signal integrity that will prepare graduates to work in this field. In fact, none of the xxx campuses offers such as course.

We have developed instructional material and delivered efficient laboratory experience with modern equipment and software tools for a signal integrity course. Modularity within the course was essential since the authors intend to infuse some of the material in existing electrical engineering courses such as digital courses, signal and systems, digital signal processing (DSP), Radio Frequency (RF) and microwave courses within the existing Penn State university curriculum. Moreover, the authors envision integration of a the signal integrity course with the extensive electrical connector business in the Central Pennsylvania area by producing graduates with hands-on experience in signal integrity.

This paper is organized as follows: In Section 2, we present the course goals. In Section 3, the course contents and detailed syllabus is presented. Section 4 describes initial results and comments from students. In section 5, conclusion and further work are detailed.

\section{Course goals}

The topic of signal integrity has been long identified ${ }^{1-4}$ as a key area for scientific development as the speed of new digital designs are pushed into the gigabit range. According to the NSF workshop report ${ }^{1}$ of 1998: "Future integrated circuit designs will be driven by interconnect, not transistors. Furthermore, interconnect technology is changing rapidly. As a result, it is critical to develop methodological, modeling, and algorithmic methods which can handle future generations of interconnect. Signal integrity is a major problem today and will become more of a problem. New interconnect technologies, such as copper and low-temperature interconnect, will introduce new problems." Therefore, interconnect performance has became one of the key factors in enabling reliable system operation at these high speeds. In order to ensure reliable operation at high data rates, signal integrity has to be addressed ${ }^{1-8}$. Signal integrity means that the signal is unimpaired with regard to its functionality ${ }^{2}$. In a system with long interconnects, there are several elements that can degrade the signal such as coupling capacitances, ground 
capacitances, mutual inductances, self-inductances, and wire resistances. These parasitic elements can produce data losses, crosstalk, jitter, and time delays that can significantly degrade system performance and reliability.

In the Harrisburg metropolitan area, the "Connector Capital of the World," has one the highest concentration of electrical connector companies in the world. These companies deal with designing and producing interconnects for very high transmission rates. The overall economic impact in the Central Pennsylvania Area is estimated at fifteen billion dollars ${ }^{13-16}$. To compete nationally and internationally, these companies rely on advances in interconnect technology. In several outreach technical meetings with EE faculty members, the engineers of these companies have indicated that it would be very important for them if there were local support for retraining their workforce, and to prepare future students to work in this field. Therefore, the authors have adopted a systematic approach to meet this need by developing a course and laboratory in signal integrity that prepare graduates to work in this field, support local industry in their research and development, and provide training through continuing education which will also benefit national and local companies to attain a well-trained workforce. In addition, this will enhance the university's outreach activities in the local community.

Another challenge that engineering educators are facing today is the fast-paced change in technology overall and communications systems in particular. Thus, the engineering faculty is faced with the need to not only prepare the students for today's job market, but also to prepare them for a lifelong learning experience. The rapidly advancing technologies are directing educators to guide students to become more actively involved in the learning process and to direct them to create synthesis skills. The current teaching paradigm in engineering education is mostly lecture/recitation, with knowledge acquisition as the main objective. One of the problems with this approach is that students are passive in the learning process and retain only a small amount of knowledge. The students find themselves quickly forgetting what they have learned, and what they do learn quickly becomes old information due to today's fast changing technology. Therefore, it is very important for engineering students to develop synthesis thinking skills so they can adapt to fundamental changes in communication technology in general, and in signal integrity in particular. Hence, the authors nurture collaborative learning in a laboratory setting.

One of the other highly interesting points in signal integrity is that it is inherently multidisciplinary. It involves several fields within the electrical engineering discipline, such as microwave, DSP, RF, very large scale integration (VLSI) and signal processing, as well as other fields such as modeling, stamping, molding and contact physics which are in the realm of mechanical engineering or physics. Therefore students must be aware of how these disciplines interplay within the analysis and design of working inter-connect prototypes.

To meet the needs described in the previous paragraphs, the authors developed and implemented a course on signal integrity. The course provides students with a theoretical background that addresses some of the important problems in signal integrity. Additionally, laboratory experiences allow students to perform hands-on experiments that facilitate their understanding of major signal integrity issues such as impedance mismatch, jitter, near-end and far-end crosstalk, time delays, equalization, de-embedding and general measurement techniques. Students are exposed to equipment and software that allow them to simulate, analyze and verify the described 
signal integrity problems using Time Domain Reflectometer, Vector Network Analyzer, highend Bit Error Tester and 3D field solvers and current signal integrity software tools. The course was offered as special topics (EE 497). The course will be submitted for official approval by the academic committees within the Penn State University system. Once the approval is finalized the course will be incorporated in the curriculum as one of the technical elective courses for the EE program at Penn State Harrisburg.

The following general set of outcomes were designed for this course:

- Demonstrate knowledge as to why signal integrity is important in today's high speed environment

- Demonstrate the ability to use modern simulation tools to predict high frequency device behavior and validate the simulation results with measurements using up to date instruments

- Design the appropriate experiment(s) to obtain meaningful high frequency data, including the need for plane definition and de-embedding

- Demonstrate the ability to work collaboratively towards finding solutions to signal integrity problems.

In addition, the author invited a well-known engineer in the SI field to discuss practical applications in class.

\section{Course Description and syllabus}

Below is the course description, which include laboratory experiences. The textbook was chosen after extensively investigating and inquiring opinions from working signal integrity engineers. The textbook "Signal and Power Integrity - Simplified (2nd Edition)" is one of the most used by industry, with very high rating by technical readers.

\section{EE 497A INTRODUCTION TO SIGNAL INTEGRITY FOR INTERCONNECTS (4)}

Catalog Data: Transmission lines and reflections, lossy lines, rise time, material properties, cross talk in transmission lines. Time and frequency domain measurements, jitter. Prerequisite: E E 330, E E 352. (Note 3 hour lecture and 3 hour lab per week)

Eric Bogatin, Signal and Power Integrity - Simplified (2nd Edition), Textbook Prentice Hall, 2009.

References: $\quad$ 1. H. Johnson and M. Graham, High-Speed Digital Design: A Handbook of Black Magic, Prentice Hall 1993.

2. D. Brooks, Signal Integrity Issues and Printed Circuit Board Design, Prentice Hall, 2003.

3. S. Hall, G. Hall and J. McCall High Speed Digital Design: A Handbook for Interconnect Theory and Design Practices, Wiley IEEE Press 2000.

$\begin{array}{llr}\text { Grading } & 2 \text { Exams } & 50 \% \\ \text { Policy: } & \text { Labs } & 15 \%\end{array}$




$\begin{array}{lc}\text { Final Project } & 15 \% \\ \text { Homework } & 10 \% \\ \text { Quizzes } & 10 \%\end{array}$

\begin{abstract}
Computer MATLAB, PSpice, and Electromagnetic field solver will serve as the Usage software platforms for this course. Students need to get their computer accounts activated in the EE computer lab.
\end{abstract}

\title{
OUTLINE
}

This outline is a guide to the topics to be covered this semester. Additional reading materials may be provided. Minor changes to this outline may occur through the semester.

\section{Introduction to Signal Integrity}

Definitions, What is signal Integrity? Signal quality on a single net; cross talk. Creating circuit models; the role of measurements.

\section{Time and Frequency Domain}

Brief review of Fourier Transform, spectrum of a repetitive signal, effect of bandwidth on rise time.

\section{The Physical Basis or Resistance and Capacitance}

Bulk resistivity, resistance per length, sheet resistance, current flows in capacitors, power and ground planes and decoupling capacitance, capacitance per length. 2D solvers.

\section{The Physical Basis of Inductance}

Partial inductance, effective inductance, total or net inductance, and ground bounce, loop inductances, current distribution and skin depth, Eddy currents. 2D model examples of inductance circuits.

\section{The Physical Basis of Transmission lines}

Uniform transmission line, instantaneous impedance of a transmission line. Driving a transmission line, return paths. Characteristic impedance and controlled impedance. Frequency variation of the characteristic impedance. Calculation of the characteristic impedance with a 2D filed solvers. S-parameters, Smith chart, and S-parameters measurements.

\section{Transmission Lines and Reflections}

Reflection at impedance changes, reflections from resistive loads. Source impedance. Bounce diagrams. Simulating reflected waveforms. Measuring reflections with TDR. Reflections from short series transmission lines, short-stub transmission lines and capacitive end termination. Effects of corners and vias. Loaded lines.

\section{Lossy lines, Rise Time Degradation and Material Properties}

Losses in transmission lines, the dissipation factor, modeling lossy transmission line. Signal velocity in a lossy transmission line. The bandwidth of an interconnect. Time domain behavior of 
lossy lines. Improving the eye diagram of transmission line. Pre-emphasis and equalization. Modeling, simulation, verification, and testing of lossy line examples.

\section{Cross Talk in Transmission Lines}

Origin of coupling, cross talk in transmission lines: NEXT and FEXT, describing cross talk. The Maxwell capacitance matrix and 2D field solvers, the inductance matrix. Near-end cross talk. Farend cross talk. Decreasing Far-End cross talk.

\section{De-embedding}

Basic Concepts of De-embedding. Different de-embedding methods. Phase uncertainty and error analysis.

\section{Partial List of Laboratory Experiments}

1. Why Signal Integrity is Important

2. Using a Simulation Tool to Visualize Time and Frequency Domain Signals

3. Transmission Line Modeling

4. The Time Domain Reflectometer (TDR) as a fundamental tool in Signal Integrity

5. Impedance and Time Delay of a Transmission Line

6. Understanding Cross Talk and De-skewing Differential Signaling

7. Using A Vector Network Analyzer to Measure S-parameters

8. Designing a Microstrip Line Using HFSS

9. Using MATLAB Scripts for S-parameter analysis

10. Understanding Communication Channel Behavior by Observing an Eye Diagram

11. Eye Diagram and Jitter Measurement Using BERT and DCA

The authors strongly believe that a signal integrity course must include meaningful hand-on experiences with equipment most likely to be found in the industry. Fortunately, the Center for Signal Integrity, at Penn State Harrisburg is equipped for the electrical design and testing of high speed interconnects. All aspects of circuit design, connector design, data acquisition and management of these associated processes are carried out on one of the lab's four computer workstations. Each station runs a variety of CAD programs, including SPICE, Advanced Design System (ADS), Ansoft HFSS, MATLAB, and PLTS. These machines are networked, have full Internet access and print to the lab's color laser printer. The lab has one Tektronix DSA8200 Digital Serial Analyzer Time Domain Reflectometer (TDR) capable of 4 port single ended and 2port differential measurement. This instrument also runs Tektronix I-Connect software capable of extracting frequency domain data from time domain measurements. The Center recently acquired a new Tektronix DSA8300. In addition, the lab has one Agilent E8364B four-port network analyzer with an Agilent N4693 E-cal Module capable of measurements up to $50 \mathrm{GHz}$. The authors recently purchased a Bit Error Rate Tester (BERT) and Digital Communication Analyzer (DCA). There are also a variety of cables, adapters, and probes for measurement of high speed devices. Test devices include several SMA type PCB boards for measurement and validation of computer modeling, and Altera's FPGA boards. Equipment and software for this laboratory have been acquired with funding from industry/state partners. At this point, this laboratory is primarily used for faculty, master, and undergraduate research in signal integrity. As it is well-known, high frequency equipment is also high priced. Most of the equipment 
described above was obtained by authors through external funding, most recently with a Major Research Instrumentation (MRI) grant from the National Science Foundation (NSF).

\section{Results}

Part of this course was piloted for engineers from local connector companies as a short one-week course. The course received very good feedback, through surveys, from the engineers participating in the course. The authors decided to deploy an academic version of the course during fall of 2015 and 2016 at xxx as an elective 400-level (senior standing) course. There were 12 students enrolled in fall 16. As culminating experience, the students were required to work on a final project, submit a report and present their work to the class. Below, is a list with title and short description of three projects:

\section{CAT 6 Cable Testing}

In this project students performed (see also fig. 1) the following:

- Calibration with a kit up to $26 \mathrm{GHz}$ for a 4-port precision network analyzer (PNA),

- Measure s-parameter and save it in ".s4p" format,

- Use ".s4p" files in MATLAB to compare NEXT \& FEXT measurements between middle pair to the rest 4 pairs and itself,

- Demonstrate differential values such as; impedance, return loss, insertion loss,

- Demonstrate common values; impedance, return loss, insertion loss.

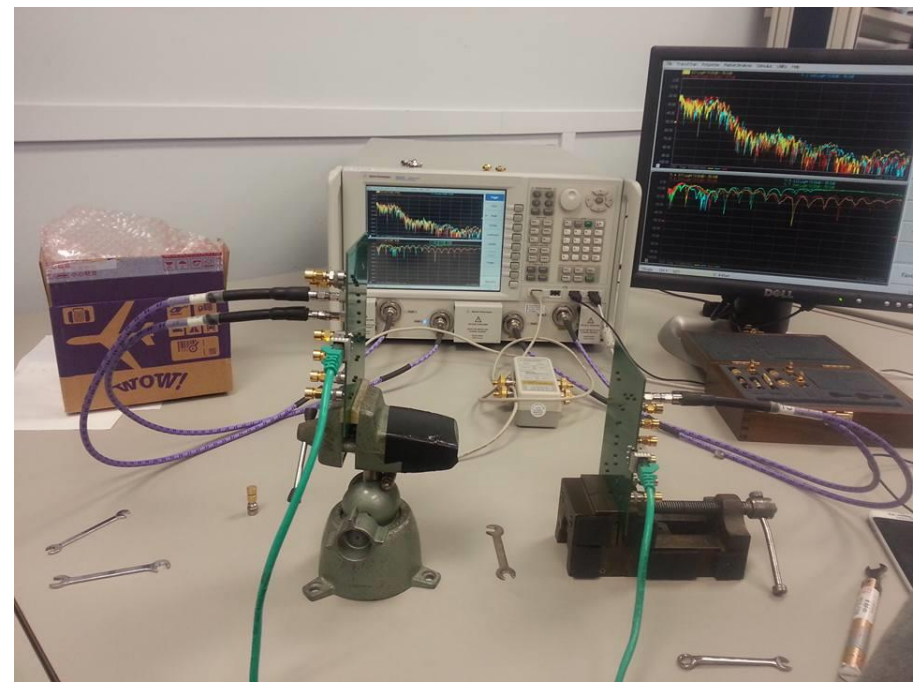

Fig. 1: Testing pairs for both ends of the cable for FEXT measurements

\section{Signal Integrity Problems with the Non-Mating Connector (NMC) and HFSS Simulations}

The scope of this project is to perform a simulation of an NMC concept for the USB 3.0 using HFSS. The objective is to obtain a plot of differential insertion loss from the NMC model, and map those results on the USB 3.0 standard (see Fig. 2). The simulations were carried out as a 
proof-of-concept proposal because of the unique characteristics of insolation from environmental effects, such as ambient moisture.

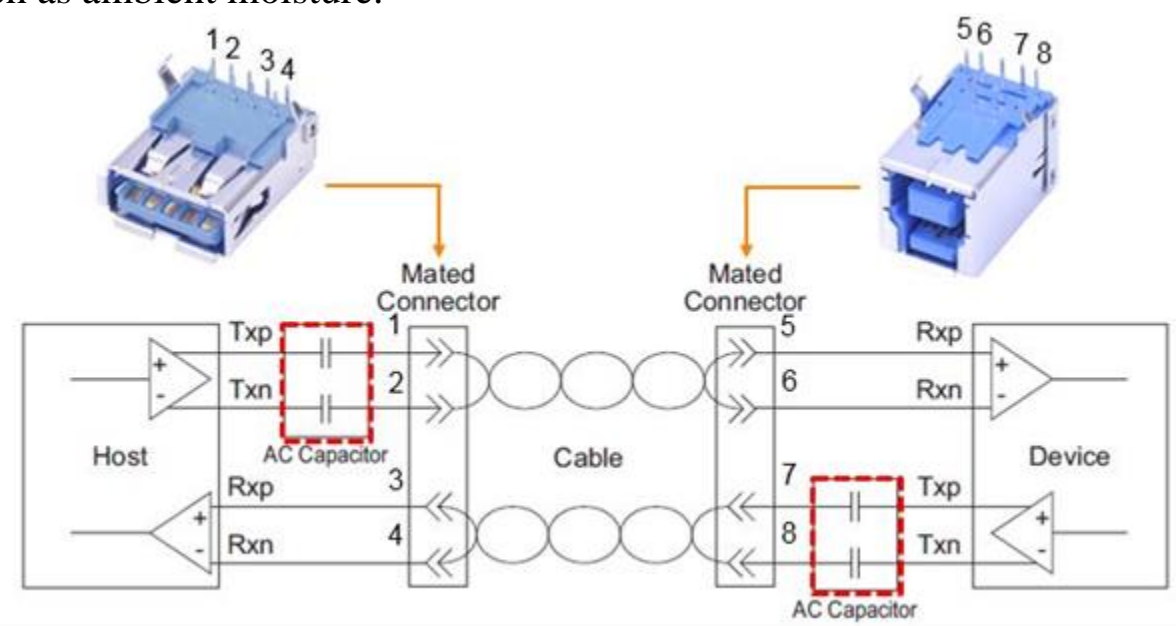

Fig. 2: USB 3.0 channel model showing two differential signals with AC bypass capacitors (as part of the NMC Connector in red) in the signal pathway ${ }^{17}$.

\section{Effects on Non-linearity on Jitter using a Keysight J-BERT and DCA-X 86100D Wide Band Oscilloscope}

The purpose of this project is to understand one of the important signal integrity topics: jitter and how to measure it using the BERT-Bit Error Ratio Tester and the DCA-X oscilloscope. Jitter is a problem that degrades system performance by generating bit errors at the receiver side. Types of jitter, causes of jitter and jitter measurement techniques are discussed in this report. In the lab the BERT is connected to the Oscilloscope through short ISI channels and jitter measurement is done on the Oscilloscope by looking at the eye diagram and the bathtub diagram. The software M8070A is used to set up the clock and the input signals. In addition, DCA-X 86100D was MATLABprogrammed for Jitter measurement when a non-linearity is present in the receiver (see Fig. 3).

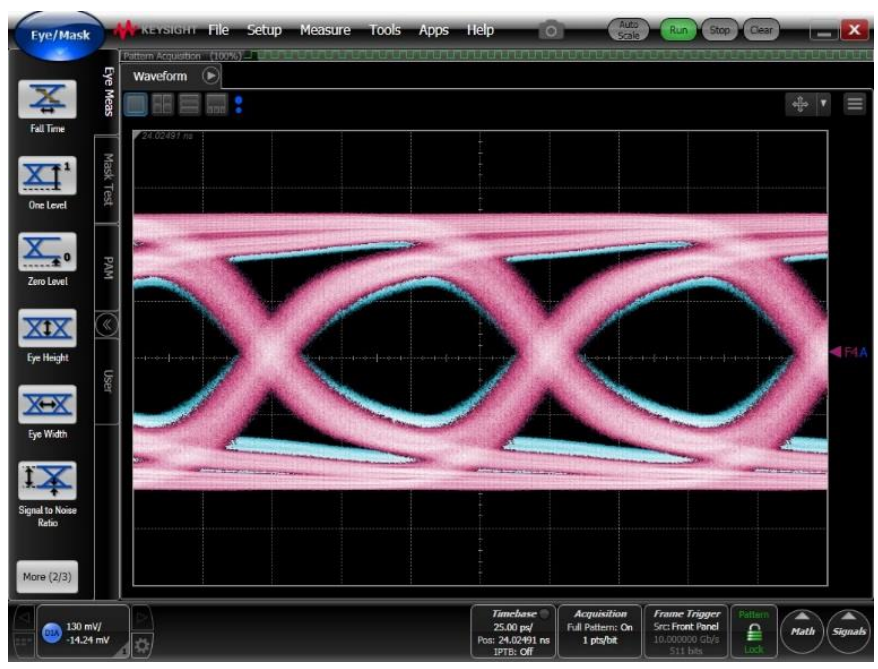

Fig. 3: Screen shot of the DCA showing the differential effect of the nonlinear (blue) and linear signal conditioning (pink). 


\section{Study of Jitter in High Speed PAM 4}

The purpose of this project is to understand the PAM 4 systems and issues involved with it. The Keysight J-BERT, along with a Keysight arbitrary wave generator were used to measure jitter from the eye diagram in PAM 4. The issues involved with the impairments created in the eye diagram for PAM 4 systems were also discussed. Although the industry has not come to an agreement to what jitter in PAM 4 means, nevertheless, students did investigate some of these issues. Fig 4. shows a screen shot of the DCA, depicting PAM 4 eye diagram, while Fig. 5 shows measuring of jitter at a crossing in the eye diagram.

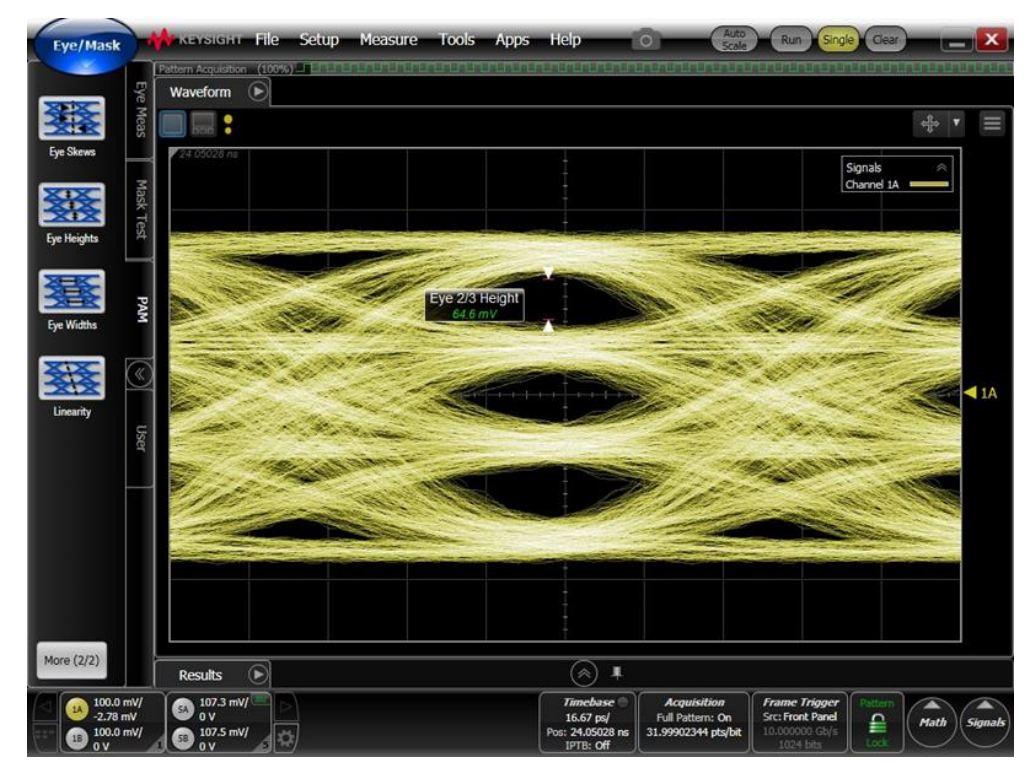

Fig. 4: Eye diagram in a PAM 4 system

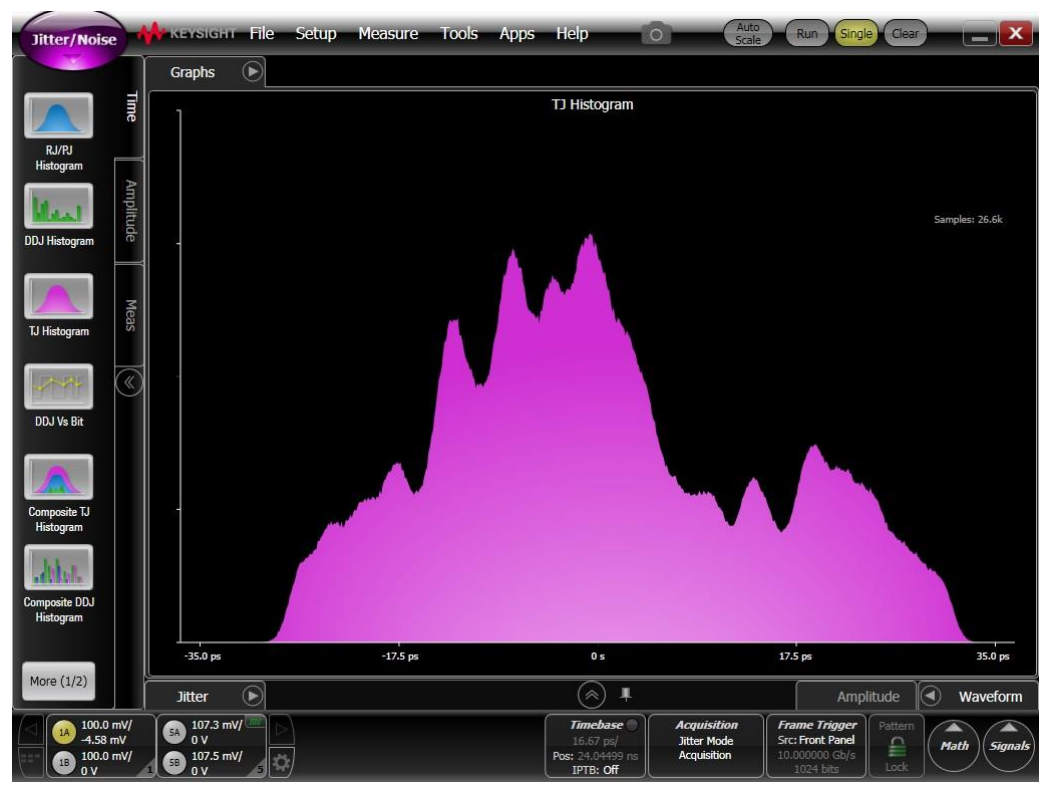

Fig. 5: Total jitter in PAM 4 system. 
At the end of the semester, the authors evaluated the course using the following survey:

\section{Evaluation Comment Sheet}

Listed below are questions to give you an opportunity to comment on the effectiveness of this signal integrity course. Your feedback is crucial to improve the delivery/content/duration of the lecture and lab components of the course.

1. Did you have exposure to signal integrity (SI) before?

2. Is the textbook practical enough to understand the concepts?

3. Do you feel that you learned the tools (software and hardware) to understand SI concepts?

4. Rate the usefulness of the laboratory experiments (1 to 5) with 5 being the highest value

5. What did you like best about this course?

6. What did you like least about this course?

7. What suggestions do you have for improving this course?

Based on the above survey, students were very satisfied with the course and laboratory contents. There were also valid suggestions, like, for instance, shorting some topics while deepening the discussion in others. There was also a suggestion to broaden the scope of the homework problems. In addition to the above survey, the university has a standard tool to measure the effectiveness of a course and its delivery, called Student Rate of Teaching Effectiveness (SRTE's). Feedback from the SRTEs was highly positive on the course contents, delivery and specifically the hand-on experiences. For instance in the SRTEs, there are two crucial questions (depicted below) graded from 1 to 7 (with 7 being the top score)

\begin{tabular}{|c|c|c|c|c|c|c|c|c|c|c|c|c|}
\hline A3 & $\begin{array}{l}\text { Rate the } \\
\text { overall } \\
\text { quality of } \\
\text { this course. }\end{array}$ & $0 / 0 \%$ & $0 / 0 \%$ & $0 / 0 \%$ & $0 / 0 \%$ & $0 / 0 \%$ & $3 / 43 \%$ & $4 / 57 \%$ & 7 & 6.57 & 0 & 123456 \\
\hline A4 & $\begin{array}{l}\text { Rate the } \\
\text { overall } \\
\text { quality of the } \\
\text { instructor. }\end{array}$ & $0 / 0 \%$ & $0 / 0 \%$ & $0 / 0 \%$ & $1 / 14 \%$ & $1 / 14 \%$ & $1 / 14 \%$ & $4 / 57 \%$ & 7 & 6.14 & 0 & 123 \\
\hline
\end{tabular}

The quality of instruction and the instructor are rated 6.57 and 6.14 (out of seven), respectively. Sample of answers to open ended questions were depicted below.

\section{University Open Ended Items}

\begin{tabular}{|l|l|}
\hline $\begin{array}{l}\text { Open } \\
\mathbf{1}\end{array}$ & What helped you learn in this course? \\
\hline Instructors genuinely love the subject. \\
\hline Paying attention in the lectures and applying theoretical effects to measurement \\
\hline Examples and the lab \\
\hline
\end{tabular}


the challenge of new concepts and material

Open What changes would improve your learning?

Can't think of any.

more hands on experience with the new equipment

Unrestricted Items

\begin{tabular}{|l|l}
\hline $\mathbf{E} \mathbf{E}$ & What did you like least about this course? \\
$\# \mathbf{2}$ &
\end{tabular}

Honestly, I feel the lab reports are unnecessary. We write enough lab reports in other classes, and I feel lab reports are not really needed in this course.

Nothing.

From the group that took the course during Fall 15, 5 students were hired mostly for the knowledge they acquired on the signal integrity course.

\section{Conclusions}

Based on their research emphasis, the authors developed and implemented a course on signal integrity during Fall 15 and Fall 16. The course provided students with a theoretical and practical background that addresses some of the important problems in signal integrity. The authors empathize that the hands-on laboratory are very important for the students to fully understanding the major signal integrity issues such as impedance mismatch, jitter, near-end and far-end crosstalk, time delays, equalization, de-embedding and general measurement techniques. Students were exposed to equipment and software that allow them to simulate, analyze and verify the described signal integrity problems using Time Domain Reflectometer, Vector Network Analyzer, high-end Bit Error Tester and 3D field solvers and current signal integrity software tools. The authors also invited a known-guest speaker to provide an industry's view of signal integrity. In addition, the authors presented some of the projects performed by students. Students' comments and ratings were very high. Students also posed suggestions that authors fully intend to address as future work. The authors note that students who took the course were sought after by industry. A follow up graduate course is also part of the authors' agenda.

\section{References}

[1] Final Report: NSF Workshop on Billion-Transistor Systems found in Princeton University website in http://www.princeton.edu/ wolf/nsf-workshop/final-report.html.

[2] A. Morales and S. Agili "Signal Integrity Challenges," Editorial Page, Ingeniare. (Chilean Journal of Engineering) Revista Chilena de Ingeniería, vol. $17 \mathrm{~N}^{\circ}$ 1, January-April, 2009.

[3] L. Green, "Understanding the Importance of Signal Integrity," Circuits and Devices, November 1999.

[4] D. Smolyansky , "Signal Integrity Key for Gbit Interconnects," EE Times, December 8, 2003

[5] B. Young, Digital Signal Integrity, Modeling and Simulation with Interconnects and Packages, Prentice Hall, 2001. 
[6] Eric Bogatin, Signal and Power Integrity - Simplified (2nd Edition), Prentice Hall, 2009.

[7] H. Johnson and M. Graham, High-Speed Digital Design: A Handbook of Black Magic, Prentice Hall 1993.

[8] D. Brooks, Signal Integrity Issues and Printed Circuit Board Design, Prentice Hall, 2003.

[9] JianJian Song, Deborah Walter and Edward Wheeler, "Rationales on a Required Class on Signal and Power Integrity in a Computer Engineering Curriculum," Proceedings of the ASEE Conference 2013.

[10] E. Wheeler, D. Voltmer, and J. Song, "Effective Integration Of Electromagnetic Compatibility And Signal Integrity In Electrical And Computer Engineering Curricula," Proceedings of 2006 Annual Conference \& Exposition, Chicago, Illinois. https://peer.asee.org/265

[11] Christopher T. Allen, "Development of a Course on High-Speed Digital Circuit Design," Proceedings of the 2003 ASEE Midwest Section Meeting, University of Missouri-Rolla, 2003.

[12] Andrew Rusek, Michelle Merrifield, Subramaniam Ganesan, "Linking Industrial Research Projects and Education," Proceedings of the 2015 ASEE North Central Section Conference, University of Cincinnati, April 17-18, 2015.

[13] Bishop \& Associates Report, connector industry market researcher. Address: 1209 Fox Glen, Dr., St Charles, Ill., 60174.

[14] Fleck's report, http://www.fleckresearch.com/reports.htm

[15] https://www.samtec.com/

[16] http://www.jst.com/home4.html

[17] Joshua S. Benjestorf, Non-Mating Connector, Patent US 20140120747 A1. 